\title{
CASE REPORT OF A SUCCESSFUL PREGNANCY IN A CYSTIC FIBROSIS PATIENT WITH THE c.1521_1523delCTT/c.3718-2477C > T GENOTYPES
}

Spasova $\mathrm{VL}^{1, *}$, Koleva $\mathrm{LI}^{2}$, Toncheva $\mathrm{DI}^{1}$, Karamisheva $\mathrm{VI}^{3,4}$

"Corresponding Author: Victoria L. Spasova, M.D., Department of Medical Genetics, Medical University of Sofia, 2 Zdrave Str., 1431 Sofia, Bulgaria. Tel: +359-887-06-65-87. Fax: +359-9520-357.

E-mail: vic.spasova@gmail.com

\begin{abstract}
The aim of this case report was to show the consequences of pregnancy in a cystic fibrosis $(\mathrm{CF})$ patient with a rare mutation. We present a case of a patient with $\mathrm{CF}$, pregnant for the second time, who gave birth to a healthy child. Her mutation status revealed the presence of relatively rare mutation c. $3718-2477 \mathrm{C}>\mathrm{T}$ that is associated with a milder phenotype of the disease. During pregnancy, her vital signs were within normal limits. She had no exacerbations after the third gestational month. Cystic fibrosis is the most common genetic disorder among Caucasians. Over the last few decades, the survival rate and the lifespan of patients with CF have increased progressively. This is why more affected women are choosing to become pregnant. Predictive factors for the pregnancy outcome are basal pulmonary function [measured by forced expiratory volume/1 second (FEV1)], nutritional status [measured by body mass index (BMI)], diabetes and bacterial colonization. The report of our case emphasizes the need for establishing the exact mutations in CF patients who plan to become pregnant in order to predict the possible outcomes of this specific period of life. Moreover, genetic counseling is strongly recommended for the right understanding of the pregnancy risks in such cases.
\end{abstract}

Keywords: Cystic fibrosis (CF); Genetic status; $\mathrm{Mu}$ tation; Pregnancy.

\footnotetext{
${ }^{1}$ Department of Medical Genetics, Medical University of Sofia, Bulgaria

2 Operative Gynaecology Clinic, UMBALSM “N. I. Pirogov”, Sofia, Bulgaria

${ }^{3}$ Second Gynaecology Clinic, SBALAG "Maichin dom", Sofia, Bulgaria

${ }^{4}$ Department of Obstetrics and Gynaecology, Medical University of Sofia, Bulgaria
}

\section{INTRODUCTION}

Cystic fibrosis $(\mathrm{CF})$ is a genetic disorder inherited in an autosomal-recessive manner. It is caused by a mutation in the CFTR gene, which codes for a chloride transporter found on the surface of the epithelial cells that line the exocrine glands. Reduced chloride conductance results in thick secretions that damage the respiratory system, pancreas, reproductive system and hepatobiliary ducts. Cystic fibrosis is the most common inherited disease in Caucasian populations, with an incidence of $1 / 2500$ newborns [1]. Cystic fibrosis carrier frequency in Bulgaria is $1 / 33$, meaning that $1 / 3600$ newborns are affected [2].

\section{CASE REPORT}

Our patient is a 30 -year-old woman who had been suffering minor signs of the disease, infections and bronchial obstruction during infancy, with the diagnosis of CF not being confirmed until the age of 20 . Genetic testing revealed she is a carrier of two different mutations: c.1521_1523 delCTT and c.3718-2477C $>$ T. After genetic counseling, our patient's partner was also tested. The CFTR panel for $95.0 \%$ of disease-relevant mutations in Bulgarian patients was used. No mutations were found.

The symptoms of the patient include permanent cough with variable expectorations. She has at least two exacerbations a year that present with increased cough, increased dyspnea, fever, change in sputum, hemoptysis, laboratory and radiographic changes indicative of pulmonary infection. A wide variety of respiratory system changes occurred since the initiation of symptoms, drumstick fingers with clubbing of the nails, hyper-resonance at percussion, enlarged anterio-posterior diameter of the thorax, diminished vesicular murmur bilaterally, variable 
rales, and hemoptysis during menstruation. In 2008, she received diagnosis of chronic maxillary sinusitis and early stage bronchiectasis.

As of 20 years of age, our patient has had a chronic infection with Staphylococcus aureus and Pseudomonas aeruginosa. In 2012, a second strain of $P$. aeruginosa was isolated. In addition, $H$. influenza, A. baumannii, $S$. maltophilia, and some less pathogenic bacteria, were also isolated from sputum. This led to treatment with wide range of injectable and oral antibiotics selected after antibiogram. As Aspergillus fumigatus was isolated from sputum samples, a long course of Variconasol had been added as part of the therapy. In 2006, she started permanent therapy with tobramycin inhalation solution $\left(\mathrm{TOBI}^{\circledR}\right.$ ) (for periods of 28 days on/off) that later was changed to tobramycin inhalation powder (TIP). From the beginning of 2016, she has been on Colistin therapy (for periods of 1 month on/off).

Through the years, structural changes in the lungs became more apparent on CT scan and X-rays, interstitial fibrotic tissue, noticeable bronchiectasis and signs of hyperventilation. Although our patient had many exacerbations through the years, lung function remains stable [forced expiratory volume/1 second (FEV1) 92.0-99.0\%, forced vital capacity (FVC) 93.0-105.0\%). Liver and pancreas function are comparatively conserved and her nutrition status is satisfactory body mass index [(BMI) 19.36]. She had some complaints of gastro-esophageal reflux. Patient regularly takes Pancreatin, Sylimarin and Esomeprazole. Her blood glucose is within normal range.

Our patient got pregnant for the first time in 2014. The pregnancy was terminated due to the long-term tobramycin treatment. Aminoglycosides are considered potentially ototoxic and nephrotoxic to the fetus and their use is an indication for pregnancy termination.

Her second pregnancy was planned. At the time she got pregnant she had been on therapy with Dornase alpha, Colistin, and Acetylcysteine. Variconasol treatment was terminated 3 months before pregnancy. At 10 weeks' gestation she was admitted to Pulmology Clinic at the Acibadem City Clinic, Tokuda Hospital, Sofia, Bulgaria, due to increased coughing, green sputum, fatigue, and rhinitis. S. aureus was cultured predominantly from the sputum sample. The therapy was prescribed after antibiogram. Amoxicillin/Clavulanic acid (1000 mg/8 hours for 14 days), Azithromycin (500mg /24 hours for 3 days, Cefuroxime (1.5 g/8 hours for 14 days), Dornase alpha, Colistin and Acetylcysteine. She was discharged from the hospital after 7 days in stable condition. This was her last exacerbation (December 2017). Her lung function remained stable during pregnancy (FEV1 $>90.0 \%$ ).

She gave birth at term through a planned Cesarean section to a healthy child in June 2018. The patient and her child were in good condition immediately after birth. No postpartum complications were registered. The mother and the baby were discharged from the hospital after 5 days.

\section{DISCUSSION}

In this case report, we document a case of a woman suffering from CF with genotype c.1521_1523delCTT/ c.3718-2477C $>$ T, who became pregnant for the second time. Monitoring of pregnancy in such cases is a great challenge because of the lack of experience of the obstetricians. According to the Cystic Fibrosis Foundation, the pregnancy rate, worldwide, in women with $\mathrm{CF}$ was reported to be 273 in 2017. Moreover, a literature review revealed some possible complications concerning both mother and child. In 1960, the first successful pregnancy of a woman with CF was reported [3]. The baby was delivered prematurely and healthy, but the mother died from lung failure 5 weeks later. Nowadays, the life expectancy of CF patients is approximately 30 years [4]. Increased life expectancy, new personalized treatment and better quality of life lead to more women with $\mathrm{CF}$ reaching childbearing age and choosing to become pregnant.

Prematurity is one of the most common complications in pregnant women with CF [5,6]. Until now, there have been no reports of higher rates of congenital malformations in babies born to mothers with CF compared to those born to healthy mothers [7], despite the possible teratogenic effect due to extensive drug therapy. Women with CF who became pregnant, frequently experience gestational diabetes, hemoptysis and reduced pulmonary function [8]. Interestingly, our patient had none of these as her only hospitalization was for a pulmonary infection and was successfully treated with a combination of antibiotics.

Different factors have been supposed to predict the outcome of the pregnancies of CF patients, pre pregnancy FEV1 $[9,10]$, pre pregnancy nutrition status $[11,12]$, medication usage [13], CF-related diabetes [14,15]. Moreover, pre pregnancy FEV1 is significantly associated with gestational age at delivery [8].

The most common mutation in the CFTR gene is c. 1521 1523delCTT, which is found in $83.0 \%$ of Bulgarian muco-viscidosis patients, as in $43.0 \%$ of them, it is in combination with other mutation [2]. The frequency of c.3718-2477 C>T (legacy name $3849+10 \mathrm{kbC}>\mathrm{T}$ ) mutation in Bulgarian patients is $1.92 \%$ [2]. Studies show that patients with the c.3718-2477C $>\mathrm{T}(3849+10 \mathrm{kbC}>\mathrm{T})$ mutation are diagnosed at a more advanced age and are in a better nutritional state [16]. This mutation causes the creation of an alternative splice acceptor site. Thus, abnormal mRNA is produced but normally spliced mRNA also occurs. This is why the c. $3718-2477 \mathrm{C}>\mathrm{T}(3849+10 \mathrm{kbC}>\mathrm{T})$ mutation is associated with milder phenotype [17]. 
In conclusion, lung function is crucial for the longterm prognosis of pregnant women suffering from CF. Poor lung function can cause pregnancy complications and worsening symptoms of the disease. On the contrary, stable lung function before and during pregnancy, ensures a good pregnancy outcome and fewer exacerbations during this period. Even though CF is a well-studied disease, managing pregnant patients with the disease is still a great challenge for obstetricians and the more information is provided the better.

As the lifespan of CF patients increases, the possibility of women getting pregnant and giving birth is much more likely. Counseling women with CF willing to become pregnant is essential for the right understanding of possible complications, as well as for a better outcome for both mother and baby.

Declaration of Interest. The authors report no conflicts of interest. The authors alone are responsible for the content and writing of this article.

Funding. This study was supported by the Bulgarian Ministry of Education and Science under the National Program for Research "Young Scientists and Postdoctoral Students."

\section{REFERENCES}

1. Davies JC, Alton EWFW, Bush A. Cystic fibrosis. Br Med J. 2007; 335(7632): 1255-1259.

2. Savov A. Genetic defects in patients with cystic fibrosis in Bulgaria. InSpiro. 2011; 3(15): 30-33.

3. Siegel B, Siegel S. Pregnancy and delivery in a patient with cystic fibrosis of the pancreas: Report of a case. Obstet Gynecol. 1960; 16(4): 438-440.

4. UK Cystic Frbrosis Registry, 2015. Cistyc fibrosis strength in numbers. Annual Data Report, 2014; 1-60.

5. Hilman BC, Aitken ML, Constantinescu AM. Pregnancy in patients with cystic fibrosis. Clin Obstet Gynecol. 1996; 39(1): 70-86.

6. ØDegaard I, Stray-Pedersen B, Hallberg K, Haanaes OC, Storrøsten OT, Johannesson M. Maternal and fe- tal morbidity in pregnancies of Norwegian and Swedish women with cystic fibrosis. Acta Obstet Gynecol Scand. 2002; 81(8): 698-705.

7. Goddard J, Bourke SJ. Cystic fibrosis and pregnancy. Obstet Gynaecol. 2009; 11(1): 19-24.

8. Renton M, Priestley L, Bennett L, Mackillop L, Chapman SJ. Pregnancy outcomes in cystic fibrosis: A 10-year experience from a UK centre. Obstet Med. 2015; 8(2): 99-101.

9. Edenborough FP, Borgo G, Knoop C, Lannefors L, Mackenzie WE, Madge S, et al. Guidelines for the management of pregnancy in women with cystic fibrosis. J Cystic Fibros. 2008; 7(Suppl 1): S2-S32.

10. Goss CH, Rubenfeld GD, Otto K, Aitken ML. The effect of pregnancy on survival in women with cystic fibrosis. Chest. 2003; 124(4): 1460-1468.

11. Cheng EY, Goss CH, McKone EF, Galic V, Debley $\mathrm{CK}$, Tonelli MR, et al. Aggressive prenatal care results in successful fetal outcomes in CF women. J Cystic Fibros. 2006; 5(2): 85-91.

12. Michel SH, Mueller DH. Nutrition for pregnant women who have cystic fibrosis. J Acad Nutrition Dietetics. 2012; 112(12): 1943-1948.

13. Geake J, Tay G, Callaway L, Bell SC. Pregnancy and cystic fibrosis: Approach to contemporary management. Obstet Med. 2014; 7(4): 147-155.

14. Cousins L. Etiology and Prevention of Congenital Anomalies Among Infants of Overt Diabetic Women. Clin Obstet Gynecol. 1991; 34(3): 481-493.

15. Hardin DS, Rice J, Cohen RC, Ellis KJ, Nick JA. The metabolic effects of pregnancy in cystic fibrosis. Obstet Gynecol. 2005; 106(2): 367-375.

16. Augarten A, Kerem B, Yahav Y, Noiman S, Rivlin Y, Tal A, et al. Mild cystic fibrosis and normal or borderline sweat test in patients with the $3849+10 \mathrm{kbC}(\mathrm{R}) \mathrm{T}$ mutation. Lancet. 1993; 342(8862): 25-26.

17. Ozdemir TR, Kanik A. A case of cystic fibrosis with a rare mutation $(3849+10 \mathrm{kbC}>\mathrm{T})$ and normal sweat chloride levels. Iranian J Pediatr. 2015; 25(2): e369-e369. 